\title{
ERRATUM
}

\section{Functional characterization of the rod visual pigment of the echidna (Tachyglossus aculeatus), a basal mammal- ERRATUM}

CONSTANZE BICKELMANN, JAMES M. MORROW, JOHANNES MÜLLER, AND BELINDA S.W. CHANG

doi:10.1017/S0952523812000223, Published by Cambridge University Press 9 July 2012.

The publisher apologizes for the following error to the Supplementary Materials that accompany this article.

For the article by Bickelman et al., published online 9th July 2012, the wrong file was posted for Bickelmann supplementary table 3. The correct file for Bickelmann supplementary table 3 has been uploaded to replace it.

\section{Reference}

Bickelmann et al., Functional characterization of the rod visual pigment of the echidna (Tachyglossus aculeatus), a basal mammal. Visual Neuroscience Published by Cambridge University Press, 9 July 2012. doi:10.1017/S0952523812000223 